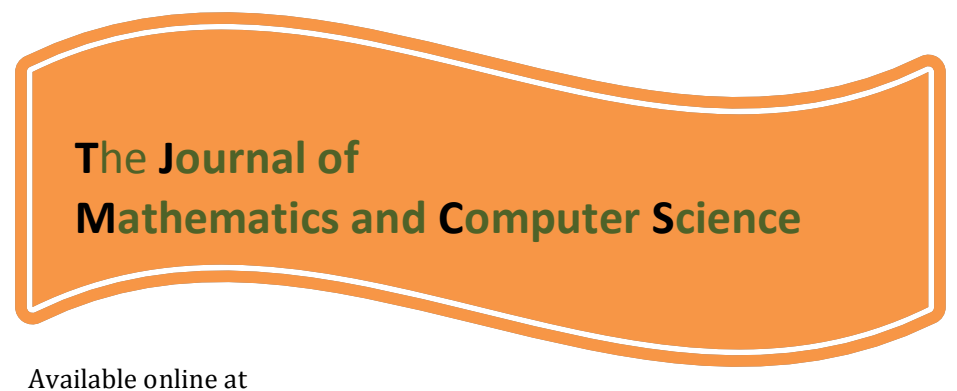

\title{
http://www.TIMCS.com
}

The Journal of Mathematics and Computer Science Vol. 4 No.1 (2012) 25 - 31

\section{A New Approach to Find All Solutions of Fuzzy Nonlinear Equations}

\author{
H. Attari ${ }^{1}$, S.H. Nasseri ${ }^{*},{ }^{1}$, S. Chitgar ${ }^{2}$ and J. Vahidi ${ }^{3}$ \\ ${ }^{1}$ Department of Mathematics, University of Mazandaran, Babolsar, Iran \\ ${ }^{2}$ Department of Mathematics, Sharif University of Technology, Tehran, Iran \\ ${ }^{3}$ Department of Mathematics, Iran University of Sciences and Technology, Behshahr, Iran
}

Received: December 2011, Revised: March 2012

Online Publication: May 2012

\begin{abstract}
The aim of this paper is proposing a new approach for finding all solutions of system of nonlinear fuzzy equations using Fuzzy Linear Programming (FLP). This approach is based on the FLP test for nonexistence of a solution to a system of fuzzy nonlinear equations using fuzzy simplex method. Also a numerical example has proposed to show the applicability of the method.
\end{abstract}

Keywords: Fuzzy nonlinear equations, Fuzzy linear programming, Fuzzy simplex method.

\section{Introduction}

Consider the problem of finding all solutions of a system of $n$ fuzzy nonlinear equationssa

$$
\begin{gathered}
f_{1}\left(\tilde{x}_{1}, \tilde{x}_{2}, \cdots, \tilde{x}_{n}\right) \approx_{\Re} \tilde{0}, \\
f_{2}\left(\tilde{x}_{1}, \tilde{x}_{2}, \cdots, \tilde{x}_{n}\right) \approx_{\Re} \tilde{0}, \\
\vdots \\
f_{2}\left(\tilde{x}_{1}, \tilde{x}_{2}, \cdots, \tilde{x}_{n}\right) \approx_{\Re} \tilde{0},
\end{gathered}
$$

contained in a bounded rectangular region (box) $D$ in $\mathbb{F}^{n}$, where $f_{1}, f_{2}, \cdots, f_{n}$ are nonlinear fuzzy variable functions, which $f_{i}: \mathbb{F}^{n} \rightarrow \mathbb{F}, i=1, \cdots, n$ and $\mathbb{F}$ is set of all triangular fuzzy numbers. Also we assume that all coefficients of all equations are real numbers. In vector notation system (1.1) will be written as $f(\tilde{x}) \approx_{\Re} \tilde{0}$. The $\Re$ index in (1.1) denotes that the equality is based on ranking functions.

In [1] and [2] an approach has proposed to find all solutions of system of nonlinear equations in crisp environment by using a test to determine the existence and nonexistence of solutions in a box. Unfortunately, in

* Corresponding author: nasseri@umz.ac.ir 
some situations because of lack of knowledge, the parameters of the problem can't be modeled in the crisp manner. Fuzzy set theory [3] is a powerful tool for modeling uncertainty and for processing vague or subjective information in mathematical models. Here we propose an efficient method to find all solutions of the system (1.1).

In this paper, in Section 2, the basic definitions of fuzzy arithmetic and ranking functions have been given. The FLP test have been proposed and described in Section 3. Also a numerical example has been demonstrated to utilize the method in Section 4 and a short conclusion has been given in Section 5.

\section{Definitions and notations}

Here, we first give some necessary definitions and notations of fuzzy set theory [4].

\subsection{Fuzzy sets}

Definition 1: Let $\mathbb{R}$ be the real line, then a fuzzy set $A$ in $\mathbb{R}$ is defined to be a set of ordered pairs $A=$ $\left\{\left(x, \mu_{A}(x)\right) \mid x \in \mathbb{R}\right\}$, where $\mu_{A}(x)$ is called the membership function for the fuzzy set. The membership function maps each element of $\mathbb{R}$ to a membership value between 0 and 1 .

Definition 2: The support of a fuzzy set $A$ is defined as

$$
\operatorname{supp}(A)=\left\{x \in \mathbb{R} \mid \mu_{A}(x)>0\right\}
$$

Definition 3: The core of a fuzzy set is the set of all points $x$ in $\mathbb{R}$ with $\mu_{A}(x)=1$.

Definition 4: A fuzzy set $A$ is called normal if its core is nonempty. In other words, there is at least one point $x \in \mathbb{R}$ with $\mu_{A}(x)=1$.

Definition 5: The $\alpha$-cut or $\alpha$-level set of a fuzzy set $A$ is a crisp set defined by $A_{\alpha}=\left\{x \in \mathbb{R} \mid \mu_{A}(x) \geq \alpha\right\}$.

Definition 6: A fuzzy set $A$ on $\mathbb{R}$ is convex, if for any $x, y \in \mathbb{R}$ and any $\lambda \in[0,1]$, we have $\mu_{A}(\lambda x+$ $(1-\lambda) y) \geq \min \left\{\mu_{A}(x), \mu_{A}(y)\right\}$.

Definition 7: A fuzzy number $\tilde{a}$ is a fuzzy set on the real line that satisfies the conditions of normality and convexity.

Definition 8: A fuzzy number $\tilde{a}$ on $\mathbb{R}$ is said to be a triangular fuzzy number, if there exist real numbers $s, l$ and $r \geq 0$ such that

$$
\mu_{\tilde{a}}(x)=\left\{\begin{array}{cc}
1+\frac{x-s}{l}, & s-l \leq x<s, \\
1-\frac{x-s}{r}, & s \leq x<s+r \\
0, & \text { o.w. }
\end{array}\right.
$$

We denote a triangular fuzzy number $\tilde{a}$ by three real numbers $s, l$ and $r$ as $\tilde{a}=(s, l, r)$, whose meaning are depicted in Figure 1. We also denote the set of all triangular fuzzy numbers with $\mathbb{F}$.The support of $\tilde{a}$ is $(l-$ $\alpha, r+\beta)$, and the core of $\tilde{a}$ is $[l, r]$.

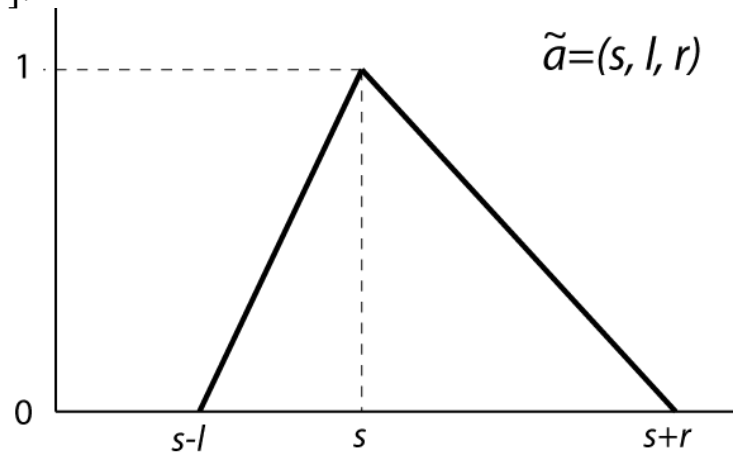

Figure 1: A triangular fuzzy number.

A real number $a$ is also a fuzzy number and it can be shown as $\tilde{a}=(a, 0,0)$. 


\subsection{Fuzzy arithmetic}

Definition 9: Let $\tilde{x}=\left(x_{1}, x_{2}, x_{3}\right)$ and $\tilde{y}=\left(y_{1}, y_{2}, y_{3}\right)$ be two triangular fuzzy numbers and $r \in \mathbb{R}$. Summation, multiplication and scalar multiplication of these fuzzy numbers defined as

$$
\begin{gathered}
r \tilde{x}=\left\{\begin{array}{cc}
\left(r x_{1}, r x_{2}, r x_{3}\right), & r \geq 0, \\
\left(r x_{1},-r x_{3},-r x_{2}\right), & r<0,
\end{array}\right. \\
\tilde{x}+\tilde{y}=\left(x_{1}+y_{1}, x_{2}+y_{2}, x_{3}+y_{3}\right), \\
\tilde{x} \tilde{y}=\left\{\begin{array}{c}
\left(x_{1} y_{1}, x_{1} y_{2}+y_{1} x_{2}, x_{1} y_{3}+y_{1} x_{3}\right), \quad \tilde{x}>\tilde{0}, \tilde{y}>\tilde{0}, \\
\left(x_{1} y_{1}, y_{1} x_{2}-x_{1} y_{3}, y_{1} x_{3}-x_{1} y_{2}\right), \quad \tilde{x}<\tilde{0}, \tilde{y}>\tilde{0}, \\
\left(x_{1} y_{1},-x_{1} y_{3}-y_{1} x_{3},-x_{1} y_{2}-y_{1} x_{2}\right), \quad \tilde{x}<\tilde{0}, \tilde{y} \prec \tilde{0} .
\end{array}\right.
\end{gathered}
$$

\subsection{Ranking functions}

Ranking is a viable approach for ordering fuzzy numbers. Various types of ranking functions have been introduced and some have been used for solving linear programming problems with fuzzy parameters. A review of some common methods for ranking fuzzy subsets of the unit interval can be seen in [5]. Here, we deal with ranking the elements of $\mathbb{F}$. In fact, an effective approach for ordering the elements of $\mathbb{F}$ is to define a ranking function $\Re: \mathbb{F} \rightarrow \mathbb{R}$ mapping triangular fuzzy numbers into $\mathbb{R}$. Consider $\tilde{a}$ and $\tilde{b}$ in $\mathbb{F}$. Define order on $\mathbb{F}$ as follows:

$$
\begin{gathered}
\tilde{a} \prec_{\Re} \tilde{b} \text { if } \Re(\tilde{a})<R(\tilde{b}), \\
\tilde{a} \approx_{\Re} \tilde{b} \text { if } \mathfrak{R}(\tilde{a})=\Re(\tilde{b}), \\
\tilde{a}>_{\Re} \tilde{b} \text { if } \Re(\tilde{a})>R(\tilde{b}) .
\end{gathered}
$$

We consider a simple linear ranking function on $\tilde{a}=(s, l, r) \in \mathbb{F}$ as:

$$
\Re(\tilde{a})=s
$$

Thus, using (2.1), for triangular fuzzy numbers $\tilde{a}=\left(s_{a}, l_{a}, r_{a}\right)$ and $\tilde{b}=\left(s_{b}, l_{b}, r_{b}\right)$, we have:

if and only if

$$
\begin{aligned}
& \tilde{a} \prec_{\Re} \tilde{b} \\
& \tilde{a} \approx_{\Re} \tilde{b} \\
& \tilde{a}>_{\Re} \tilde{b}
\end{aligned}
$$

$$
\begin{aligned}
& \left(s_{a}\right)<\left(s_{b}\right), \\
& \left(s_{a}\right)=\left(s_{b}\right), \\
& \left(s_{a}\right)>\left(s_{b}\right),
\end{aligned}
$$

respectively. Also we write $\tilde{a} \preccurlyeq_{\Re} \tilde{b}$ if $\tilde{a} \prec_{\Re} \tilde{b}$ and $\tilde{a} \approx_{\Re} \tilde{b}$ also we do for $\tilde{a} \succcurlyeq_{\Re} \tilde{b}$ in the same manner. Then, for this linear ranking function $\Re$ we may obtain: $\tilde{a} \preccurlyeq_{\Re} \tilde{b}$ if and only if $\tilde{b}-\tilde{a} \succcurlyeq_{\Re} \tilde{0}$, or if and only if $-\tilde{a} \succcurlyeq_{\Re}-\tilde{b}$.

Proposition 1: Suppose $\tilde{a}, \tilde{b}, \tilde{c}, \tilde{d}$ be triangular fuzzy numbers. If $\tilde{a} \preccurlyeq_{\Re} \tilde{b}$ and $\tilde{c} \preccurlyeq_{\Re} \tilde{d}$, then $\tilde{a}+\tilde{c} \preccurlyeq_{\Re} \tilde{b}+\tilde{d}$, also if $\tilde{a} \preccurlyeq_{\Re} \tilde{b}$ and $\tilde{c} \preccurlyeq_{\Re} \tilde{d}$, then $\tilde{a} \tilde{c} \preccurlyeq_{\Re} \tilde{b} \tilde{d}$.

Proof: Straightforward.

Remark 1: For a triangular fuzzy number $\tilde{a}$, the relation $\tilde{a} \geqslant_{\Re} \tilde{0}$ holds, if there exist $\varepsilon \geq 0$ and $\alpha \geq 0$ such that $\tilde{a} \geqslant(0, \varepsilon, \alpha)$. We realize that $\Re(0, \varepsilon, \alpha)=0$ (we also consider $\tilde{a} \approx_{\Re} \tilde{0}$ if and only if $\Re(\tilde{a})=\tilde{0}$ ). Thus, without loss of generality, throughout the paper we let $\tilde{0}=(0,0,0)$ as the zero triangular fuzzy number.

\section{FLP Test}

In this section, we first propose the solution algorithm. For the simplicity of discussion, here we assume that (1) can be presented as 


$$
f(x) \triangleq_{\Re} g(\tilde{x})+Q \tilde{x}-\tilde{r} \approx_{\Re} \tilde{0},
$$

where $g(\tilde{x})=\left[g_{1}\left(\tilde{x}_{1}\right), g_{2}\left(\tilde{x}_{2}\right), \cdots, g_{n}\left(\tilde{x}_{n}\right)\right]^{T}: \mathbb{F}^{n} \rightarrow \mathbb{F}^{n}$ is a nonlinear function with component functions $g_{i}\left(\tilde{x}_{i}\right): \mathbb{F} \rightarrow \mathbb{F}, i=1,2, \cdots, n$, and $Q$ is an $n \times n$ real constant matrix, and $\tilde{r}=\left(\tilde{r}_{1}, \tilde{r}_{2}, \cdots, \tilde{r}_{n}\right)^{T} \in \mathbb{F}^{n}$ is a fuzzy constant vector. Also we suppose that all mentioned fuzzy numbers are triangular fuzzy numbers as defined in previous section.

An $n$-dimensional fuzzy interval vector with components $\left[\tilde{a}_{i}, \tilde{b}_{i}\right], i=1,2, \cdots, n$, is denoted by

$$
X=\left(\left[\tilde{a}_{1}, \tilde{b}_{1}\right],\left[\tilde{a}_{2}, \tilde{b}_{2}\right], \cdots,\left[\tilde{a}_{n}, \tilde{b}_{n}\right]\right)^{T} .
$$

Geometrically, $X$ is a fuzzy $n$-dimensional box.

In FLP test, the nonexistence of a solution to (3.1) in $X$ is checked as follows. We first calculate the interval extensions of $g_{i}\left(\tilde{x}_{i}\right), i=1,2, \cdots, n$, over $X$. Let the interval extensions of $g_{i}\left(\tilde{x}_{i}\right)$ over $\left[\tilde{a}_{i}, \tilde{b}_{i}\right]$ be $\left[\tilde{c}_{i}, \tilde{d}_{i}\right]$. Then, we introduce auxiliary variables $\tilde{y}_{i}, i=1,2, \cdots, n$, and set $\tilde{y}_{i}=g_{i}\left(\tilde{x}_{i}\right)$. Therefore, if $\tilde{a}_{i} \preccurlyeq_{\Re} \tilde{x}_{i} \preccurlyeq_{\Re} \tilde{b}_{i}$, then $\tilde{c}_{i} \preccurlyeq_{\Re} \tilde{y}_{i} \preccurlyeq_{\Re} \tilde{d}_{i}$ holds.

Now we replace each nonlinear function $g_{i}\left(\tilde{x}_{i}\right)$ in (3.1) by the auxiliary variable $y_{i}$ and the linear inequality $\tilde{c}_{i} \preccurlyeq_{\mathfrak{R}} \tilde{y}_{i} \preccurlyeq_{\mathfrak{R}} \tilde{d}_{i}$, and we consider the following FLP problem:

max (arbitrary constant)

$$
\begin{array}{lll}
\text { s.t. } & \tilde{y}+Q \tilde{x}-\tilde{r} \approx_{\Re} \tilde{0}, & \\
& \tilde{a}_{i} \preccurlyeq_{\Re} \tilde{x}_{i} \preccurlyeq_{\Re} \tilde{b}_{i} & i=1,2, \cdots, n, \\
& \tilde{c}_{i} \preccurlyeq_{\Re} \tilde{y}_{i} \preccurlyeq_{\Re} \tilde{d}_{i}, & i=1,2, \cdots, n,
\end{array}
$$

where $\tilde{y}=\left(\tilde{y}_{1}, \tilde{y}_{2}, \cdots, \tilde{y}_{n}\right) \in \mathbb{F}^{n}$. Therefore, our problem (3.1) reduces to fuzzy linear programming problem or precisely fuzzy variable programming problem (or FVLP). Then, we can apply the fuzzy bounded simplex algorithm method [6] to equation (3.2).

Evidently, all solutions of (3.1) which exist in $X$ satisfy the constraints in (3.2) if we set $\tilde{y}_{i}=$ $g\left(\tilde{x}_{i}\right)$. Namely, the feasible region of the FLP problem (3.2) is a convex polyhedron containing all solutions of (3.1) in $X$. Hence, if the feasible region is empty, then we can conclude that there is no solution of (3.1) in $X$.

The emptiness or nonemptiness of the feasible region (3.2) can be checked by the fuzzy simplex method. If the fuzzy simplex method terminates with the information that the feasible region is empty, then there is no solution of (3.1) in $X$. We name this test as called FLP test.

The algorithm is as follows. Beginning with initial box $X=D$, the following procedure is performed recursively. At each level, we perform the FLP test on $X$. If there is no solution of (3.1) in $X$, then we exclude it from further consideration. If there are some solutions of (3.1) in $X$, then we compute them by some method. If neither existence and nor nonexistence of a solution in $X$ can be proved, the bisect $X$ in some appropriately chosen coordinate direction to form two (or more) new boxes; we then continue the above procedure with one of these boxes, and put the other one(s) on a stack for later consideration. Thus, provided the number of solutions of (3.1) contained in $D \subset \mathbb{F}^{n}$ is finite, we can find them all with mathematical certainty.

Note: The discussion of this paper is easily extendable to more general systems of nonlinear equations and more general type of fuzzy numbers.

\section{1.gniworraN PLF}

Here we propose an algorithm which is an extension of the algorithm in [2], to which the idea of narrowing a box using LP techniques is introduced. Namely, if $X$ is not excluded, then we narrow the box so that no solution is lost, which makes the algorithm much more efficient. 
We explain how $X$ is narrowed efficiently by using the FLP techniques. First, we apply the fuzzy dual simplex method [7] to

$$
\begin{array}{ccc}
\min & \tilde{x}_{i} \\
\text { s.t. } & \tilde{y}+Q \tilde{x}-\tilde{r} \approx_{\Re} \tilde{0}, & \\
& \tilde{a}_{i} \preccurlyeq_{\Re} \tilde{x}_{i} \preccurlyeq_{\Re} \tilde{b}_{i} \quad i=1,2, \cdots, n, \\
& \tilde{c}_{i} \preccurlyeq_{\Re} \tilde{y}_{i} \preccurlyeq_{\Re} \tilde{d}_{i}, \quad i=1,2, \cdots, n,
\end{array}
$$

for $i=1$. If the feasible region of (3.3) is empty, then we exclude $X$ from further consideration. If the minimum value $x_{1}^{*}$ is greater than $\tilde{a}_{1}$, then we prune the lower part $\tilde{x}_{1} \preccurlyeq_{\Re} x_{1}^{*}$ of $X$ because there is no solution there (see Fig. 2a). Note that $x_{1}^{*}$ is the lower bound of the feasible region of the LP problem (3.3) in the $x_{1}$-direction. Thus, the box $X$ is narrowed from the lower side of the $x_{1}$-direction. Let the narrowed box be denoted by $X$ again. Next, we apply the dual simplex method to (6) for $i=2$ and narrow the box from the lower side of the $x_{2}$ direction, where $\tilde{a} \preccurlyeq_{\Re} \tilde{x} \preccurlyeq_{\Re} \tilde{b}$ and $\tilde{c} \preccurlyeq_{\Re} \tilde{y} \preccurlyeq_{\Re} \tilde{d}$ represent the corresponding intervals for the new $X$ (see Fig. 2b). Of course, if the feasible region is empty, then we exclude $X$. Note that the new LP problem differs from the previously solved FLP problem only in the values of $\tilde{a}, \tilde{b}, \tilde{c}$, and $\tilde{d}$. Hence, by using the technique of sensitivity analysis, we can perform the dual simplex method beginning with the dual feasible basis which is obtained from the optimal basis of the previously solved LP problem. Let the narrowed box be denoted by $X$ again. Then, we repeat the similar procedure in the $x_{i}$-directions $(i=3,4, \cdots, n)$, and narrow the box in all coordinate directions from the lower sides or exclude the box. Let the resulting narrowed box be denoted by $X$ again. Then, we apply the fuzzy dual simplex method to

$$
\begin{array}{ccc}
\max & \tilde{x}_{i} \\
\text { s.t. } & \tilde{y}+Q \tilde{x}-\tilde{r} \approx_{\Re} \tilde{0}, \\
& \tilde{a}_{i} \preccurlyeq_{\Re} \tilde{x}_{i} \preccurlyeq_{\Re} \tilde{b}_{i} \quad i=1,2, \cdots, n, \\
& \tilde{c}_{i} \preccurlyeq_{\Re} \tilde{y}_{i} \preccurlyeq_{\Re} \tilde{d}_{i}, \quad i=1,2, \cdots, n,
\end{array}
$$

for $i=1$ on $X$. If the feasible region of (3.4) is empty, then we exclude $X$ from further consideration. If the maximum value $x_{1}$ is less than $b_{l}$, then we prune the upper part $\tilde{x}_{1} \succcurlyeq_{\Re} \tilde{x}_{1}^{*}$ of $X$ because there is no solution there (see Fig. 2c). Thus, the box $X$ is narrowed from the upper side of the $x_{1}$-direction. Let the narrowed box be denoted by $X$ again. Then, we repeat the similar procedure in the $x_{i}$-directions $i=3,4, \cdots, n$, and narrow the box in all coordinate directions from the upper sides (see Fig. 2d) or exclude the box. Such a series of procedures is called FLP narrowing.

As the box becomes smaller, the feasible region becomes smaller, which makes the FLP narrowing more and more powerful. Furthermore, the FLP problem (3.3) or (3.4) can be solved efficiently with a few iterations (often no iteration) by the dual simplex method. Thus, the FLP narrowing is not only powerful but also efficient and excludes or narrows a box effectively.

Notice that, in the FLP narrowing, we first perform the narrowing procedure from the lower sides of all $x_{i}$ directions, and then perform the narrowing procedure from the upper sides (i.e., in the order $\min x_{1}, \ldots, \min x_{n}$, $\max x_{1}, \ldots, \max x_{n}$ ). This is because it makes the number of iterations of the dual simplex method small and makes the algorithm efficient. Namely, if we perform the narrowing procedure from the lower side and that from the upper side alternately (i.e., in the order $\min x_{1}, \max x_{1}, \ldots, \min x_{n}$, max $x_{n}$ ), then the number of iterations in solving (3.4) would become large because the optimal solution of (3.3) (which is used as the initial basis in solving (3.4)) is far from the optimal solution of (3.4). We use the FLP test as the base, and perform the FLP narrowing (instead of the FLP test) for each box. If the box is not excluded, then we bisect the narrowed box in the direction where the box has the maximum width, and continue the similar procedure as that in FLP test. 
H. Attari, S.H. Nasseri, S. Chitgar, J. Vahidi / TJMCS Vol. 4 No. 1 (2012) 25 - 31
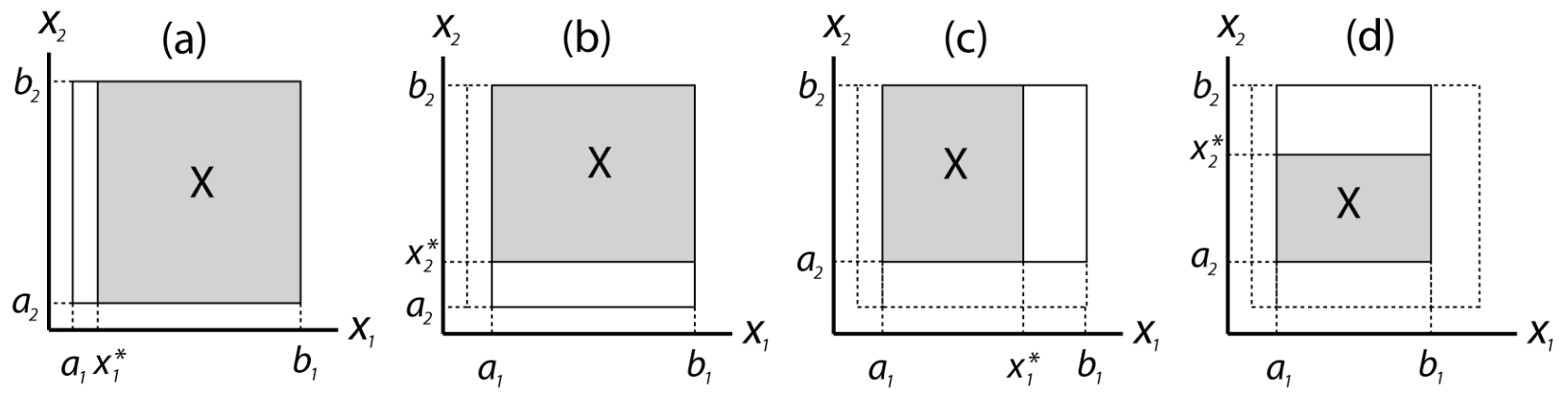

Figure 2: FLP Narrowing.

\section{Numerical Example}

Example 1: We now consider a system of $n$ nonlinear equations

$$
\tilde{x}_{i}-\frac{1}{2 n}\left(\sum_{k=1}^{n} \tilde{x}_{k}^{3}+i\right) \approx_{\mathfrak{R}} \tilde{0}, i=1,2, \cdots, n .
$$

The initial box is $D=([\tilde{0}, \tilde{2}], \cdots,[\tilde{0}, \tilde{2}])^{T}$, and all fuzzy quantities are supposed symmetric triangular fuzzy numbers with same left and right spreads like as $(s, 1,1)$. The following table shows the computation time of the proposed algorithm. $n$ and s denote the number of equation and the number of solutions in $D$. We implemented the proposed algorithm using the Wolfram Mathematica by Java language on an Intel Core 2 Duo P8700 2.53 GHz, 4 GB RAM.

\begin{tabular}{ccc}
\hline$n$ & S & Times $(\mathrm{s})$ \\
\hline 3 & 2 & 0.9 \\
5 & 2 & 8 \\
10 & 2 & 29 \\
\hline
\end{tabular}

\section{Conclusion}

In this paper, an efficient algorithm has been proposed for finding all solutions of systems of nonlinear fuzzy equations using FLP test. The proposed algorithm is efficient and has the possibility of solving large-scale systems of nonlinear equations in practical computation time. The interesting feature of this algorithm is that FLP problems per each box actually can be solved efficiently by the fuzzy dual simplex method. Thus, the proposed algorithm seems to be a useful tool for finding all solutions of nonlinear equations.

\section{Acknowledgments}

The authors express their gratitude to Research Center of Algebraic Hyperstructures and Fuzzy Mathematics, Babolsar, Iran, for its partially support.

\section{References}

[1] K. Yamamura, "Finding all solutions of nonlinear equations using linear combinations of functions", Reliable Comput., Vol. 6, pp.105-113, 2000. 
H. Attari, S.H. Nasseri, S. Chitgar, J. Vahidi / TJMCS Vol. 4 No. 1 (2012) 25 - 31

[2] K. Yamamura, S. Suda and N. Tamura, "LP narrowing: A new strategy for finding all solutions of nonlinear equations", Applied Mathematics and Computation, Vol. 215, pp. 405-413, 2009.

[3] L.A. Zadeh, "Fuzzy sets", Information and Control, Vol. 8, pp. 338-353, 1965.

[4]G.J. Klir and B. Yuan, "Fuzzy Sets and Fuzzy Logic: Theory and Applications", Prentice-Hall, PTR, New Jersey., 1995.

[5] X. Wang and E. Kerre, "Reasonable properties for the ordering of fuzzy quantities (2 parts)", Fuzzy Sets and Systems, Vol. 118, pp. 375-405, 2001.

]6] A. Ebrahimnejad, S.H. Nasseri and F. Hosseinzadeh-Lotfi, "Bounded linear programs with trapezoidal fuzzy numbers", International Journal of Uncertainty, Fuzziness and Knowledge-Based Systems, Vol. 18, No. 3, pp. 269-286, 2010.

17] N. Mahdavi-Amiri and S.H. Nasseri, "Duality results and a dual simplex method for linear programming problems with trapezoidal fuzzy variables”, Fuzzy Sets and Systems, Vol. 158, pp. 1961-1978, 2007. 